Letter to the Editor

\section{Positive symptoms and social cognition impairment affect severity of suicidal ideation in schizophrenia}

\section{Dear Editor,}

Recently, the Danish OPUS study (Madsen et al., 2016) demonstrated that suicidal ideation, when present at first episode of psychosis, persists during the first 10 years after treatment initiation, thus fitting as a stable clinical feature and representing an early indicator of poor global outcome. We aimed to investigate the nature of suicidal ideation as a stable trait of schizophrenia through the study of its relations with other stable features of the disorder.

Among 86 clinically stable Caucasian outpatients aged 18 and older (mean age 36.3; SD 10.8; 74\% males; duration of illness 12.4 years SD 9.5) with an established diagnosis of schizophrenia, we examined the relationship between the severity of suicidal ideation and (1) symptoms assessed through a five factor model of the PANSS; (2) neurocognitive domains assessed by the Measurement and Treatment Research to Improve Cognition in Schizophrenia (MATRICS) Battery; and (3) global functioning through the GAF. According to Mosolov et al. (2012), patients were defined as clinically stable when they demonstrated no change $>1$ point for any item of the positive subscale of the PANSS over the six months prior to the study. On the basis of the presence $(\mathrm{SI}+)$ or absence $(\mathrm{SI}-)$ of suicidal ideation (defined as thoughts of engaging in suicidal behavior with or without a definitive plan, but with no evidence of current suicidal behavior), we categorized patients into two groups (respectively 38 vs 48 participants). Severity of lifetime suicidal ideation was assessed with the Columbia Suicide Severity Rating Scale "Lifetime version" (Posner et al., 2011).

A comparison of the two groups demonstrated that the SI + group was more impaired on psychopathological and neurocognitive variables than the SI - group (Table 1), whereas age, gender, education, or duration of illness did not differ significantly between the groups.

In the SI + group, SI severity was significantly related to positive symptoms $(r=0.637, p<0.001)$, excitement $(r=0.364, p<0.05)$, disorganization $(r=0.360, p<0.05)$, social cognition impairment $(r=$ 0.329, $p<0.05)$ and global functioning $(r=-0.527, p<0.001)$. A stepwise regression analysis showed that the positive symptom domain and social cognition explained $44.1 \%$ of variance of the severity of SI (Table 2).

Overall, our results indicated that the SI + group reported more severe symptoms, impairment of functioning, and had higher neurocognition compared to the SI -, suggesting a possible bi-directional interpretation. Namely, SI severity may be interpreted as an illness-related indicator of poor global outcome, or vice versa, the latter may generate profound dissatisfaction with life and lead to SI.

In our study, the finding that SI severity contributes to poorer outcomes was confirmed by its strong relationship with global functioning impairment and psychotic positive symptoms. Of note, suicidal ideation is associated with psychotic positive symptoms even when they are present at a subthreshold level (Kelleher et al., 2012; DeVylder et al., 2015).

Our results confirm the influence of positive symptoms on suicidal ideation and also revealed a novel finding with regard to the effect of social cognition on SI. This is a very important finding considering that social cognition impairment has been recognized as an endophenotype of schizophrenia, related both to neurodevelopmental and to genetic risk (Kohler et al., 2010; Lavoie et al., 2013). As a marker of vulnerability, social cognition impairment is present in prodromal subjects, remains stable through the course of the illness (Comparelli et al., 2013), and is detectable among child and adolescent offspring of parents with schizophrenia (Horton et al., 2017). Importantly, in a recent study, the researchers found that among adolescents following psychiatric hospitalization, emotion dysregulation, including non-acceptance of emotional responses and more limited access to emotion regulation strategies, differentiated chronic suicidal ideation from declining suicidal ideation (Wolff et al., 2017).

The main implication of these findings is that suicidal ideation among patients with schizophrenia may emerge from the interplay of disturbed emotion management with aberrant dopaminergic firing underpinning positive symptoms. Both social information and aberrant salience processing disturbance may have a downstream effect on an individual's ability to function in everyday life and lead to poor interpersonal functioning.

Based on the fact that the severity of suicidal ideation is related to poor functioning and influenced by a stable trait of the illness, it should be considered beyond the risk of suicide itself, and recognized as a severity index of the disorder, irrespective of related suicidal behavior.

Unfortunately, the cross-sectional design of our study does not allow us to draw conclusions regarding different trajectories of suicidal ideation through the course of the illness; thus, future longitudinal research is needed to confirm this hypothesis.

Finally, our study underscores the crucial role of social and emotional cognition contributing to the course and the outcome of schizophrenia and, hopefully, highlights new perspectives for the prevention of suicide.

\section{Conflict of interest}

Authors of this paper have no relevant affiliations or financial involvement with any organization or entity with a financial interest in, or financial conflict with the subject matter or materials discussed in the manuscript. This includes employment, consultancies, honoraria, stock ownership or options, expert testimony, grants or patents received or pending, or royalties. No writing assistance was utilized in the production of this manuscript.

\section{Contributors}

Anna Comparelli designed the study, wrote the protocol, and revised the final draft of the manuscript.

Valentina Corigliano wrote the first draft of the manuscript.

Dorian A. Lamis approved the final manuscript.

Antonella De Carolis undertook the statistical analysis. 


\section{Table 1}

Comparison between patients without suicidal ideation $(\mathrm{SCH}-\mathrm{SI})$ and patients with suicidal ideation $(\mathrm{SCH}+\mathrm{SI})$ on socio-demographics, psychopathological and neurocognitive measures.

\begin{tabular}{|c|c|c|c|c|c|}
\hline & \multirow{2}{*}{$\begin{array}{l}\mathrm{SCH}-\mathrm{SI} \\
(n=48) \\
\mathrm{N}(\%)\end{array}$} & \multirow{2}{*}{$\begin{array}{l}\mathrm{SCH}+\mathrm{SI} \\
(n=38) \\
\mathrm{N}(\%)\end{array}$} & \multicolumn{3}{|c|}{ Analyses } \\
\hline & & & $\chi^{2}$ & d.f. & $p$ \\
\hline \multirow[t]{2}{*}{ Male } & $35(73 \%)$ & $27(71 \%)$ & 0.037 & 1 & 0.848 \\
\hline & Mean (SD) & Mean (SD) & $\mathrm{F}$ & d.f. & $p$ \\
\hline Age (years) & $35.9(10.6)$ & $36.8(11.3)$ & 0.5 & 84 & 0.721 \\
\hline Years of education & $12.9(2.9)$ & $12.8(3.2)$ & 0.2 & 84 & 0.865 \\
\hline Duration of illness (years) & $11.2(8.9)$ & $13.9(10.1)$ & 0.9 & 84 & 0.204 \\
\hline $\mathrm{GAF}^{* *}$ & $45.6(8.4)$ & $38.7(9.6)$ & 0.3 & 84 & 0.000 \\
\hline PANSS POS* & $9.4(3.9)$ & $11.3(4.7)$ & 3.4 & 84 & 0.044 \\
\hline PANSS NEG* & $16.1(6.4)$ & $19.1(6.9)$ & 0.7 & 84 & 0.042 \\
\hline PANSS DIS* & $16.1(6.4)$ & $8.9(3.6)$ & 4.1 & 84 & 0.041 \\
\hline PANSS EXC & $8.6(3.8)$ & $9.4(3.6)$ & 0.1 & 84 & 0.320 \\
\hline PANSS DEP** & $5.5(2.6)$ & $8.9(3.8)$ & 12.7 & 84 & 0.000 \\
\hline Speed of processing* & $0.15(0.8)$ & $-0.19(0.7)$ & 0.4 & 84 & 0.037 \\
\hline Sustained attention/vigilance & $0.06(0.7)$ & $-0.08(1.3)$ & 0.1 & 84 & 0.526 \\
\hline Working memory* & $0.19(0.9)$ & $-0.24(0.8)$ & 1.1 & 84 & 0.029 \\
\hline Verbal learning & $0.15(0.9)$ & $-0.18(1.0)$ & 0.8 & 84 & 0.129 \\
\hline Visual learning & $0.12(1.1)$ & $-0.15(0.8)$ & 3.1 & 84 & 0.210 \\
\hline Reasoning and problem solving & $0.14(1.0)$ & $-0.18(0.9)$ & 0.9 & 84 & 0.137 \\
\hline Social cognition & $-0.06(0.9)$ & $0.09(1.0)$ & 1.1 & 84 & 0.496 \\
\hline
\end{tabular}

* $p<0.05$.

*** $p<0.001$.

Table 2

Stepwise multiple regression in predicting severity of suicidal ideation $(n=38){ }^{\mathrm{a}}$

\begin{tabular}{llllll}
\hline Step & Variable & B & Sig. & R-square & Adjusted R-square \\
\hline 1 & Positive symptoms & 0.166 & 0.000 & 0.367 & 0.349 \\
2 & Social cognition & -0.470 & 0.015 & 0.473 & 0.441 \\
\hline
\end{tabular}

${ }^{\text {a }}$ Dependent variable: severity of suicidal ideation; independent variable: positive symptoms, excitement symptoms, disorganized symptoms, global assessment functioning, social cognition.

Eleonora De Pisa interpreted data.

Lorenzo Stampatore managed the literature searches and analyses.

Paolo Girardi and Maurizio Pompili revised the final manuscript.

\section{Acknowledgements}

We are grateful to all subjects who participated in this study.

\section{References}

Comparelli, A., Corigliano, V., De Carolis, A., Mancinelli, I., Trovini, G., Ottavi, G., Dehning, J., Tatarelli, R., Brugnoli, R., Girardi, P., 2013. Emotion recognition impairment is present early and is stable throughout the course of schizophrenia. Schizophr. Res. 143 (1): 65-69. http://dx.doi.org/10.1016/j.schres.2012.11.005.

DeVylder, J.E., Thompson, E., Reeves, G., Schiffman, J., 2015. Psychotic experiences as indicators of suicidal ideation in a non-clinical college sample. Psychiatry Res. 226 (2-3), 489-493.

Horton, L.E., Bridgwater, M.A., Haas, G.L., 2017. Emotion recognition and social skills in child and adolescent offspring of parents with schizophrenia. Cogn. Neuropsychiatry 22 (3):175-185. http://dx.doi.org/10.1080/13546805.2017.1297223.

Kelleher, I., Lynch, F., Harley, M., Molloy, C., Roddy, S., Fitzpatrick, C., Cannon, M., 2012. Psychotic symptoms in adolescence index risk for suicidal behavior: findings from 2 population-based case-control clinical interview studies. Arch. Gen. Psychiatry 69 (12):1277-1283. http://dx.doi.org/10.1001/archgenpsychiatry.2012.164.

Kohler, C.G., Walker, J.B., Martin, E.A., Healey, K.M., Moberg, P.J., 2010. Facial emotion perception in schizophrenia: a meta-analytic review. Schizophr. Bull. 36 (5), 1009-1019.
Lavoie, M.A., Plana, I., Bédard Lacroix, J., Godmaire-Duhaime, F., Jackson, P.L., Achim, A.M. 2013. Social cognition in first-degree relatives of people with schizophrenia: a metaanalysis. Psychiatry Res. 209 (2), 129-135.

Madsen, T., Karstoft, K.I., Secher, R.G., Austin, S.F., Nordentoft, M., 2016. Trajectories of suicidal ideation in patients with first-episode psychosis: secondary analysis of data from the OPUS trial. Lancet Psychiatry. 3 (5), 443-450.

Mosolov, S.N., Potapov, A.V., Ushakov, U.V., 2012. Remission in schizophrenia: results of cross-sectional with 6-month follow-up period and 1-year observational therapeutic studies in an outpatient population. Ann. General Psychiatry 11 (1), 1.

Posner, K., Brown, G.K., Stanley, B., Brent, D.A., Yershova, K.V., Oquendo, M.A., Currier G.W., Melvin, G.A., Greenhill, L., Shen, S., Mann, J.J., 2011. The Columbia-Suicide Severity Rating Scale: initial validity and internal consistency findings from three multisite studies with adolescents and adults. Am. J. Psychiatry 168 (12), 1266-1277.

Wolff, J.C., Davis, S., Liu, R.T., Cha, C.B., Cheek, S.M., Nestor, B.A., Frazier, E.A., Schaffer, M.M. Spirito, A., 2017. Trajectories of suicidal ideation among adolescents following psychiatric hospitalization. J. Abnorm. Child Psychol. (Mar 27) http://dx.doi.org/10.1007/ s10802-017-0293-6.

Anna Comparelli

Department of Neurosciences, Unit of Psychiatry, Sapienza University of Rome, School of Medicine and Psychology, Via di Grottarossa 1035-39,

00198 Rome, Italy

Corresponding author.

E-mail address: anna.comparelli@uniroma1.it.

Valentina Corigliano

Department of Neurosciences, Unit of Psychiatry, Sapienza University of Rome, School of Medicine and Psychology, Via di Grottarossa 1035-39,

00198 Rome, Italy

Dorian A. Lamis

Department of Psychiatry and Behavioral Sciences, Emory School of Medicine, Grady Hospital, Atlanta, Georgia

Antonella De Carolis

Department of Neurosciences, Unit of Neurology, Sapienza University of Rome, School of Medicine and Psychology, Via di Grottarossa 1035-39,

00198 Rome, Italy

Lorenzo Stampatore

Department of Neurosciences, Unit of Psychiatry, Sapienza University of Rome, School of Medicine and Psychology, Via di Grottarossa 1035-39,

00198 Rome, Italy

Eleonora De Pisa

Department of Neurosciences, Unit of Psychiatry, Sapienza University of Rome, School of Medicine and Psychology, Via di Grottarossa 1035-39,

00198 Rome, Italy

Paolo Girardi

Department of Neurosciences, Unit of Psychiatry, Sapienza University of Rome, School of Medicine and Psychology, Via di Grottarossa 1035-39, 00198 Rome, Italy

Maurizio Pompili

Department of Neurosciences, Unit of Psychiatry, Sapienza University of Rome, School of Medicine and Psychology, Via di Grottarossa 1035-39, 00198 Rome, Italy

18 April 2017

Available online $\mathrm{xxxx}$ 